\title{
Evolution of lithium beyond the solar age: A Li survey of the old open cluster NGC $188^{\star}$
}

\author{
S. Randich ${ }^{1}$, P. Sestito ${ }^{2}$, and R. Pallavicini ${ }^{3}$ \\ 1 INAF/Osservatorio Astrofisico di Arcetri, Largo E. Fermi 5, 50125 Firenze, Italy \\ 2 Dipartimento di Astronomia, Università di Firenze, Largo E. Fermi 5, 50125 Firenze, Italy \\ ${ }^{3}$ INAF/Osservatorio Astronomico di Palermo, Piazza del Parlamento 1, 90134 Palermo, Italy
}

Received 1 August 2002 / Accepted 27 November 2002

\begin{abstract}
We have determined Li abundances for $11 \mathrm{G}$-type stars in the 6-8 Gyr old open cluster NGC 188 . These data significantly enlarge the number of cluster stars with Li measurements, allowing us to extend the investigation of Li depletion in open clusters to ages well beyond the age of the Sun. We have also inferred the cluster metallicity which turns out to be solar. We find that solar-type stars in NGC 188 are only slightly more Li depleted than the much younger Hyades and no more Li depleted than stars of similar temperature in the 2-4 Gyr younger cluster M 67. At variance with M 67, NGC 188 members show virtually no scatter in their Li abundances. Surprisingly, no solar-type star in NGC 188 appears as Li depleted as the Sun or as the most Li depleted stars in M 67. We discuss the implications of these results for mechanisms of internal mixing and $\mathrm{Li}$ depletion in main sequence stars.
\end{abstract}

Key words. stars: abundances - stars: evolution - open clusters and associations: individual: NGC 188

\section{Introduction}

Observations of light elements in stars are powerful tools to test models of Big Bang nucleosynthesis, Galactic chemical evolution and stellar interior mixing processes. Lithium has a very low burning temperature $\left(\sim 2.5 \times 10^{6} \mathrm{~K}\right)$ and it survives only in the outermost layers of a star; therefore, it is a good tracer of mixing mechanisms and stellar interior structure. Observations of $\mathrm{Li}$ in clusters of different ages and chemical compositions allow us to put constraints on these processes and to investigate their dependence on age, metallicity and possibly other stellar parameters. Observations of cluster stars carried out during the past 15 years have shown that $\mathrm{Li}$ depletion is not a simple function of age and metallicity as predicted by standard models (see e.g. the reviews of Jeffries 2000 and Pasquini 2000 and references therein). For example, standard models, which include only convection as a mixing process, predict that little (if any) Li depletion should occur during the main sequence (MS) evolution of solar-type stars, since the convective zone is too shallow to reach the Li burning layer; the comparison of the $\mathrm{Li}$ abundance distributions of clusters of different ages (spanning the range from the zero age main sequence -ZAMS- to the solar age) indicates instead that solar-type stars do destroy $\mathrm{Li}$ on the MS. Furthermore, the solar age, solar-metallicity cluster

\footnotetext{
Send offprint requests to: S. Randich, e-mail: randich@arcetri.astro.it

^ Based on observations collected at the Italian National Telescope Galileo.
}

M 67 is characterized by a large dispersion in Li abundances for stars of similar temperatures (Spite et al. 1987; García López et al. 1988; Pasquini et al. 1997; Jones et al. 1999); about 60\% of the stars appear Li-rich, with a $\mathrm{Li}$ abundance a factor of 2-3 below the $600 \mathrm{Myr}$ old Hyades, while the remaining fraction are $\mathrm{Li}$-poor with a factor of 5-10 lower abundances. This result shows that additional parameters besides mass, age, and metallicity affect Li depletion, and that non-standard mixing processes such as diffusion, mass loss, or rotationally driven mixing, are at work.

The comparison between M 67 and the intermediate age clusters IC 4651, NGC 3680, and NGC 752 (age 2 Gyr) shows instead that stars in the upper envelope of M 67 have similar abundances to the intermediate cluster stars, suggesting that either Li depletion becomes very slow at old ages, at least for a fraction of stars, or that different conditions (e.g., heavy element abundances, initial angular momentum distributions and/or rotational evolutions) in different clusters lead to different $\mathrm{Li}$ evolutions (Randich et al. 2000). We also mention that neither IC 4651 nor NGC 3680 show any scatter in $\mathrm{Li}$, while a scatter might be present among NGC 752 members cooler than 5800 K (Hobbs \& Pilachowski 1986).

In order to put additional empirical constraints on MS Li depletion, in particular on its timescale and on the development of the dispersion, we carried out a Li survey of the old open cluster NGC 188. This cluster has an estimated age of 6-8 Gyr (see discussion in Sarajedini et al. 1999, hereafter S99) and is one of the oldest known open clusters. According to S99 


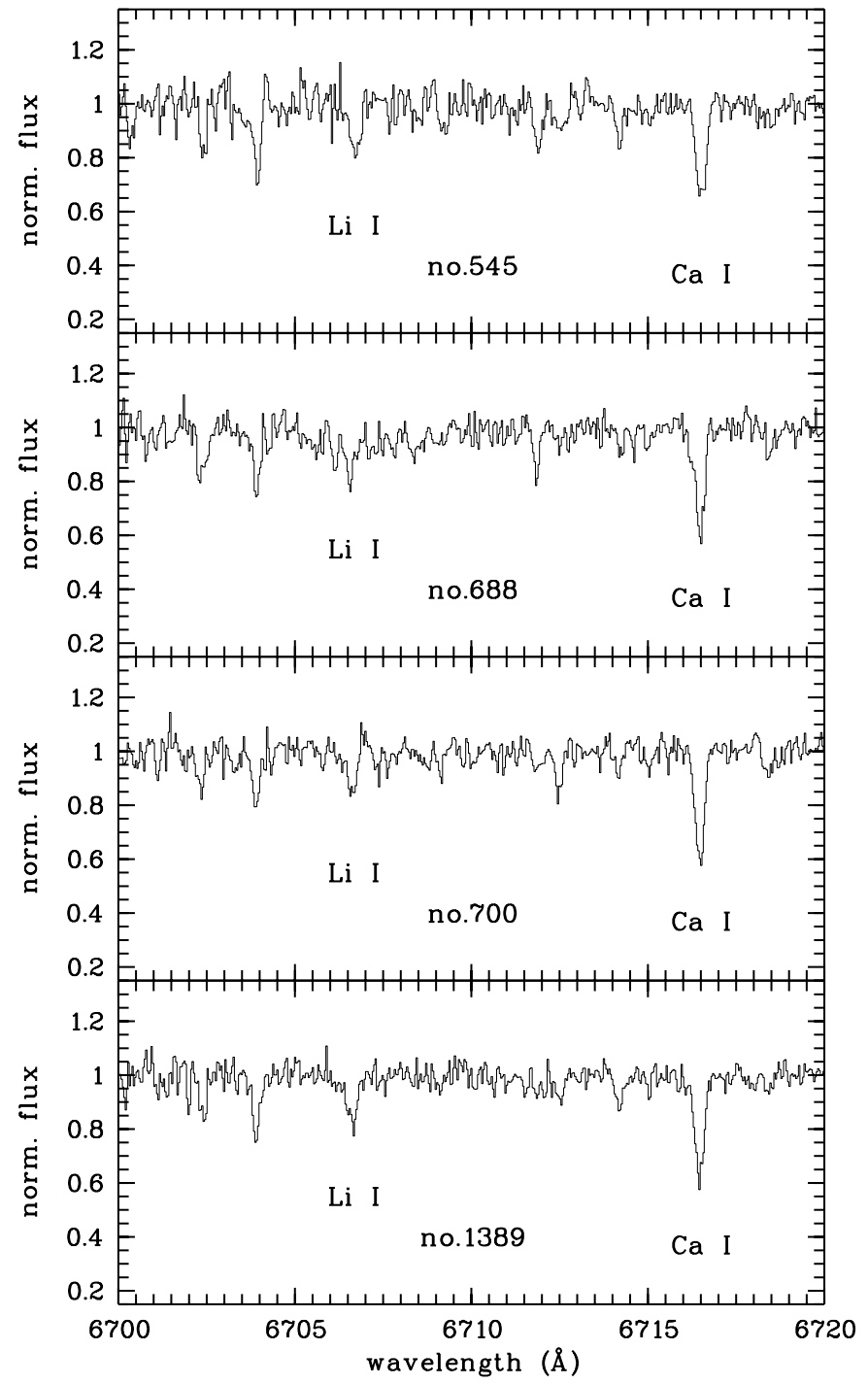

Fig. 1. Sample spectra in the Li region.

it is $3.0 \pm 0.7$ Gyr older than M 67. The only published data on $\mathrm{Li}$ abundances in dwarf stars in this cluster date back to nearly 15 yrs ago and were limited to only a few stars (Hobbs \& Pilachowski 1988). A more detailed Li study of this cluster is thus warranted. Our paper is organized as follows: in Sect. 2 we describe our sample and the observations; the abundance analysis and the results are summarized in Sects. 3 and 4, while in Sects. 5 and 6 we present a discussion of the results and our conclusions.

\section{Observations}

We selected the target stars from the proper motion study of Dinescu et al. (1996 - hereafter D96); our sample includes 11 G-type stars with membership probability larger than $90 \%$. The sample stars are listed in Table 1; in the first and second columns the numbering systems of D96 and S99 are used. In the following we will use the numbering system of D96. Three stars are in common with the study of Hobbs \& Pilachowski (1988). The observations were carried out in service mode during August 2001 at the Italian National Telescope Galileo (TNG) equipped with the SARG spectrograph
(Gratton et al. 2000). The Red Grism and the OG570 filter were used, together with the mosaic of two EEV $15 \mu$ pixel CCDs $(2048 \times 4096)$; such a combination allowed us to cover the spectral range from $\sim 550$ to $1011 \mathrm{~nm}$. $2 \times 2$ CCD binning was used. Part of the sample stars were observed using a slit width equal to $0.8^{\prime \prime}$, corresponding to a nominal resolving power $R \sim 57000$, while another fraction was observed with a larger slit $\left(1.6^{\prime \prime}\right)$. In the latter case the seeing was significantly better than the slit width and, therefore, we obtained a resolving power $R \sim 35000$ (as measured from the telluric lines), somewhat higher than the nominal one $(R \sim 29000)$.

The spectra were reduced using MIDAS in the ECHELLE context. We first separated, rotated, and flipped the two CCD; then, we carried out the reduction following the usual steps: namely, bias subtraction, order definition, order extraction, inter-order background subtraction, flat-fielding and wavelength calibration.

All the sample stars have $V$ magnitudes around 15 and all but two of them (no. 1344 and no. 700) were exposed for 2 hrs; exposure times for stars no. 1344 and 700 were slightly shorter (1.5 and $1.75 \mathrm{hrs}$, respectively). Typical $S / N$ ratios in the lithium region are in the range 20-35. The spectra of four stars in the sample are shown in Fig. 1.

\section{Abundance analysis}

With the exception of star no. 545, $B-V$ and $V$ values for all our sample stars were taken from the UBV CCD photometric study of S99; actually, since in that paper photometry for each star is not listed, we retrieved the values from the WEBDA database ${ }^{1}$. For star no. 545 we adopted the photographic photometry of D96 $(B-V=0.74, V=14.99)$, since we found significantly discrepant values in $\mathrm{S} 99(B-V=0.45, V=19.50)$ : D96 photometry is most likely the correct one, based on the spectral features and Li content of this star, which are similar to those of the other observed objects. Moreover, we would have not been able to observe an object much fainter than $V \sim 15$. Photometry for the sample stars is listed in Cols. 3 and 4 of Table 1.

Effective temperatures were determined from $B-V$ colors using the calibration of Soderblom et al. (1993). A reddening $E(B-V)=0.09$ was used (S99). A surface gravity $\log g=4.44$ was assumed for all sample stars, while microturbulence values were computed as $\xi=3.2 \times 10^{-4}\left(T_{\text {eff }}-6390\right)-1.3(\log g-$ $4.16)+1.7$ (Boesgaard \& Friel 1990). We recall that gravity and microturbulence values have very little effects on the determination of Li abundances, while they affect iron abundances. We assumed conservative random errors of $100 \mathrm{~K}$ in $T_{\text {eff }}, 0.3 \mathrm{dex}$ in $\log g$, and $0.3 \mathrm{~km} \mathrm{~s}^{-1}$ in $\xi$.

$\mathrm{Li}$ abundances were derived from measured equivalent widths of the $\mathrm{Li}$ I $\lambda 6707.8 \AA$ blend and by using the curves of growth (COG) of Soderblom et al. (1993). Li abundances were then corrected for NLTE effects using the prescriptions of Carlsson et al. (1994). We mention that at our spectral resolutions and given the slow rotation of the sample stars, we are able to separate the Li feature from the nearby Fe I $\lambda 6707.44 \AA$ line.

\footnotetext{
${ }^{1}$ http://obswww. unige.ch/webda/
} 
Table 1. Stellar parameters and Li abundances for the sample stars. Asterisks indicate objects with nominal resolving power $R=57000$.

\begin{tabular}{cccccccc}
\hline \hline $\begin{array}{c}\text { no. } \\
\text { D96 }\end{array}$ & $\begin{array}{c}\text { no. } \\
\text { S99 }\end{array}$ & $V$ & $B-V$ & $\begin{array}{c}T_{\text {eff }} \\
(\mathrm{K})\end{array}$ & $\begin{array}{c}E W(\lambda 6708 \AA) \\
(\mathrm{m} \AA)\end{array}$ & $\log n(\mathrm{Li})_{\mathrm{LTE}}$ & $\log n(\mathrm{Li})_{N \mathrm{LTE}}$ \\
\hline $545^{*}$ & 989 & 14.99 & 0.74 & 5696 & $60 \pm 7$ & 2.29 & $2.30 \pm 0.11$ \\
$612^{*}$ & 1172 & 15.05 & 0.69 & 5888 & $34 \pm 6$ & 2.19 & $2.19 \pm 0.11$ \\
$661^{*}$ & 1025 & 15.10 & 0.67 & 5967 & $64 \pm 7$ & 2.57 & $2.54 \pm 0.11$ \\
688 & 724 & 15.13 & 0.67 & 5967 & $46 \pm 7$ & 2.34 & $2.38 \pm 0.10$ \\
700 & 685 & 14.97 & 0.69 & 5888 & $40 \pm 6$ & 2.26 & $2.26 \pm 0.10$ \\
760 & 534 & 15.16 & 0.66 & 6008 & $42 \pm 6$ & 2.39 & $2.37 \pm 0.11$ \\
1344 & 1120 & 14.92 & 0.72 & 5772 & $31 \pm 6$ & 2.05 & $2.06 \pm 0.12$ \\
$1354^{*}$ & 933 & 15.05 & 0.68 & 5928 & $69 \pm 8$ & 2.58 & $2.55 \pm 0.11$ \\
1389 & 739 & 14.97 & 0.71 & 5810 & $53 \pm 7$ & 2.33 & $2.33 \pm 0.11$ \\
$1403^{*}$ & 356 & 15.06 & 0.68 & 5928 & $39 \pm 6$ & 2.29 & $2.28 \pm 0.11$ \\
$1422^{*}$ & 922 & 15.10 & 0.68 & 5928 & $49 \pm 9$ & 2.40 & $2.38 \pm 0.13$ \\
\hline
\end{tabular}

Table 2. Stellar parameters and Li abundances for the Hobbs \& Pilachowski (1988) sample.

\begin{tabular}{ccccccccc}
\hline \hline $\begin{array}{c}\text { no. } \\
\text { D96 }\end{array}$ & $\begin{array}{c}\text { no. } \\
\text { San62 }\end{array}$ & $\begin{array}{c}\text { no. } \\
\text { S99 }\end{array}$ & $V$ & $B-V$ & $\begin{array}{c}T_{\text {eff }} \\
(\mathrm{K})\end{array}$ & $\begin{array}{c}E W(\lambda 6708 \AA) \\
(\mathrm{m} \AA)\end{array}$ & $\log n(\mathrm{Li})_{\mathrm{LTE}}$ & $\log n(\mathrm{Li})_{\text {NLTE }}$ \\
\hline 661 & $\mathrm{I}-71$ & 1025 & 15.10 & 0.67 & 5967 & $40 \pm 15$ & 2.34 & $2.32 \pm 0.20$ \\
688 & $\mathrm{I}-101$ & 724 & 15.13 & 0.67 & 5967 & $40 \pm 15$ & 2.34 & $2.32 \pm 0.20$ \\
694 & 677 & $\mathrm{I}-99$ & 14.97 & 0.72 & 5772 & $72 \pm 15$ & 2.46 & $2.46 \pm 0.15$ \\
700 & $\mathrm{I}-91$ & 685 & 14.97 & 0.69 & 5888 & $25 \pm 15$ & 2.05 & $2.06 \pm 0.33$ \\
726 & $\mathrm{I}-17$ & 735 & 15.11 & 0.67 & 5967 & $40 \pm 15$ & 2.34 & $2.32 \pm 0.20$ \\
\hline
\end{tabular}

Table 3. Fe I equivalent widths and iron abundances from the two spectra obtained by adding the observed spectra of stars with similar colors. The stars used for the co-added spectra are indicated.

\begin{tabular}{ccccc}
\hline \hline \multicolumn{3}{c}{$1354+1403+1422$} & \multicolumn{2}{c}{$612+700$} \\
$\lambda(\AA)$ & $\begin{array}{c}E W \\
\end{array}$ & $\log \epsilon(\mathrm{Fe})$ & $\begin{array}{c}E W \\
(\mathrm{~m} \AA)\end{array}$ & $\log \epsilon(\mathrm{Fe})$ \\
\hline 6703.58 & 34 & 7.59 & 33 & 7.53 \\
6710.32 & 12 & 7.51 & 10 & 7.38 \\
6726.67 & 42 & 7.48 & 41 & 7.44 \\
6733.15 & 28 & 7.59 & 31 & 7.64 \\
6750.16 & 70 & 7.54 & 74 & 7.54 \\
6806.86 & 27 & 7.44 & 33 & 7.54 \\
6810.27 & 46 & 7.50 & 51 & 7.57 \\
6820.37 & 45 & 7.64 & 42 & 7.56 \\
6843.65 & - & - & 60 & 7.53 \\
average & $7.53 \pm 0.07 \pm 0.09$ & & $7.53 \pm 0.08 \pm 0.09$ \\
\hline
\end{tabular}

Starting from published EWs and colors, we re-analyzed in a consistent way the NGC 188 stars observed by Hobbs \& Pilachowski (1988). Photometric data for these stars, which are listed in Table 2 using three numbering systems (D96, San62 - Sandage 1962 and S99), were retrieved from S99. Since we want to compare the $\log n(\mathrm{Li})$ vs. $T_{\text {eff }}$ distribution of NGC 188 with both the Hyades and M 67, abundances for these two clusters were also re-determined consistently with our sample stars. Namely, for the Hyades, using colors and equivalent widths published by Balachandran (1995), we derived new $T_{\text {eff }}$ and Li abundances. Jones et al. (1999) had derived Li abundances for M 67 using the same temperature vs. color calibration and the same COGs we used in the present analysis and thus we just applied NLTE corrections to their published abundances. Errors in Li abundances for our sample stars were determined considering both errors in $E W \mathrm{~s}$ and uncertainties in effective temperatures. Measured $E W \mathrm{~s}$, effective temperatures, and derived $\mathrm{Li}$ abundances are listed in the last three columns of Table 1. The same quantities for the sample of Hobbs \& Pilachowski are listed in Table 2.

The $S / N$ ratios of our spectra are too low to allow a very accurate determination of the cluster metallicity. In order to have at least a rough estimate of the cluster $[\mathrm{Fe} / \mathrm{H}]$ from our spectra, and in particular to investigate whether or not it is significantly higher/lower than solar, we added the spectra of stars with the same colors and measured the equivalent widths of various $\mathrm{Fe}_{\mathrm{I}}$ lines in the same echelle order as lithium and in the next (towards the red) order. More specifically, we added the spectra of stars no. 1354, 1403, and $1422(B-V=0.68$, $\left.T_{\text {eff }}=5928 \mathrm{~K}\right)$ and those of stars no. 612 and $700(B-V=0.69$, $\left.T_{\text {eff }}=5888 \mathrm{~K}\right)$. We then measured the EWs of 8-9 Fe I lines, listed in Table 3, in the co-added spectra. Iron abundances were determined using MOOG (Sneden 1973 - version December 2000) and Kurucz (1995) model atmospheres. Since we wanted to carry out a differential analysis with respect to the Sun, we determined $\log g f$ values by an inverse abundance analysis of the solar spectrum (sky at twilight) obtained with SARG during our run, under the condition $\log \epsilon(\mathrm{Fe})_{\odot}=7.52$; the following solar parameters were assumed: $T_{\mathrm{eff}}=5770 \mathrm{~K}, \log g=4.44$, and $\xi=1.1 \mathrm{~km} \mathrm{~s}^{-1}$.

In Table 3 we list iron abundances for the different lines, together with the mean value for each group of stars. The error includes the standard deviation in the mean from the 
different lines (which provides an estimate of the random error due to errors in the measured equivalent widths) and the random error due to the uncertainties in stellar parameters. The latter was estimated by varying one parameter at the time and leaving the other two parameters unchanged; the errors due to each parameter were then quadratically added. Uncertainties of $\pm 100 \mathrm{~K}$ in $T_{\text {eff }}, \pm 0.3 \mathrm{dex}$ in $\log g$, and $\pm 0.3 \mathrm{~km} \mathrm{~s}^{-1}$ in $\xi$, correspond to $0.07,0.05$, and $0.02 \mathrm{dex}$ in $\log \epsilon(\mathrm{Fe})$. We find the same iron abundance for the two group of stars; the weighted mean is $\log \epsilon(\mathrm{Fe})=7.53 \pm 0.08$, i.e., NGC 188 has solar metallicity. Whereas systematic errors due e.g., to reddening, effective temperature scale, model atmospheres, atomic parameters, etc. are difficult to estimate, we note that our result is in agreement with earlier metallicity estimates based on spectroscopy (of warmer stars at the turn-off) or photometry (see discussion in von Hippel \& Sarajedini 1998).

\section{Results}

\subsection{Li equivalent widths}

In Fig. 2 we plot Li $E W$ s as a function of dereddened $B-V$ color for our sample stars (triangles) and for the sample of Hobbs \& Pilachowski (1988 -squares). Filled symbols denote the three stars in common between the two samples. The figure shows a good agreement between the $E W$ vs. color distribution of our sample stars and that from Hobbs \& Pilachowski; the $E W$ s of the stars in common are consistent within the errors, although our EWs are somewhat larger than Hobbs \& Pilachowski's. In the following analysis, therefore, we will merge the two samples, using our own Li measurements for the stars in common. The total sample contains 13 stars, 11 stars observed by us plus the two stars from Hobbs \& Pilachowski not included in our sample. Figure 2 also evidences a certain amount of star-to-star scatter. However the spread is consistent with the observational errors: the average $E W$ of our 11 measurements and of the two stars from Hobbs \& Pilachowski that are not in our sample is $49 \mathrm{m \AA}$ with a standard deviation $\sigma=13 \mathrm{~m} \AA$, that is only slightly larger than the average $E W$ error $(8 \mathrm{~m} \AA)$. In addition, as we will see in the next section, the spread in $E W$ s translates into a negligible dispersion in $\mathrm{Li}$ abundances.

\subsection{Li abundance vs. $T_{\text {eff }}$}

The usual $\log n(\mathrm{Li})$ vs. $T_{\text {eff }}$ plot is shown in Figs. 3 and 4 ; in the two figures NGC 188 is compared to the Hyades and M 67, respectively. As we have mentioned in Sect. 3, data for both clusters were retrieved from the literature and analyzed consistently with our sample stars.

The distribution of NGC 188 lies somewhat below the Hyades, but not as much as one would expect given the difference in age (a factor of at least 10). In particular, we note a couple of stars in our sample with abundances similar to those of Hyades stars with the same effective temperature. For the 13 NGC 188 stars we obtain an average Li abundance $\log n(\mathrm{Li})=2.34 \pm 0.13$; for the Hyades in the same temperature range we obtain $\log n(\mathrm{Li})=2.58 \pm 0.09$, implying a difference in Li depletion less than a factor of two.
The comparison with M 67 shows that: $a)$ the $\log n(\mathrm{Li})$ vs. $T_{\text {eff }}$ distribution for NGC 188 lies on the upper envelope of the pattern of M 67, i.e., 6-8 Gyr old members of NGC 188 are no more Li depleted than the Li-rich stars in the younger M 67; viceversa, several M 67 members are more Li depleted than NGC 188 stars. Whereas this result was already pointed out by Hobbs \& Pilachowski (1988), our observations allow us to confirm it based on a much larger sample of stars; $b$ ) NGC 188 does not show a star-to-star scatter as large as that observed among M 67 members; whereas the coolest stars in NGC 188 may be characterized by some amount of scatter, the scatter is much smaller than the dispersion of M 67 and most likely due to observational uncertainties. Our NGC 188 sample is much smaller than the M 67 sample and covers a narrower temperature interval; thus, the obvious question arises whether the lack of a significant dispersion is simply due to low number statistics and/or to the restricted temperature (mass) range. In order to address this issue, we carried out a simple statistical test; given the fraction of Li-poor stars in M 67 (we assumed a conservative value of $30 \%$, although according to Pasquini et al. 1997 it may be as high as $40 \%$ ), and assuming that the same fraction would hold also for NGC 188, we computed the probability of finding $0 / 13 \mathrm{Li}$-poor stars in NGC 188 . Using a binomial distribution, we found $P(0 / 13) \sim 9.7 \times 10^{-3}$; this means that we can exclude the possibility of the presence of Li-poor stars (and thus of a scatter) in NGC 188 at almost the $3 \sigma$ significance level. Considering a fraction Li-poor stars equal to $40 \%$, we would get a probability $P(0 / 13) \sim 1 \times 10^{-3}$ (larger than $3 \sigma$ ). In addition, even considering only stars in the temperature range covered by our NGC 188 sample, a scatter is present among M 67 members, with about $40 \%$ of the stars being Li-poor (see Fig. 4). Thus we can safely exclude that the lack of a scatter in NGC 188 is due to the narrow temperature range.

\section{Discussion}

As a summary of the results presented in the two previous sections we conclude that: 1) G-type stars in NGC 188 are no more Li depleted than Li-rich stars in M 67 and are at most a factor of two more Li depleted than their Hyades counterparts; 2) M 67 remains so far the only cluster showing a clear spread in $\mathrm{Li}$ among solar-type stars ${ }^{2}$. In other words, the evolution of the upper envelope of the Li vs. $T_{\text {eff }}$ distribution past the age of the Hyades, as well as the evolution of the spread from the age of M 67 to that of NGC 188, further challenge our understanding of the Li destruction in solar-type stars.

\subsection{The upper envelope}

As we mentioned in the introduction, Randich et al. (2000), on the basis of a comparison of the Hyades, of the intermediate age clusters IC 4651, NGC 3680, and NGC 752, and of

\footnotetext{
2 As mentioned in the introduction, NGC 752 might show a spread among stars cooler than the Sun (Hobbs \& Pilachowski 1986), but this result is based on two cluster members only and must be confirmed with a larger sample.
} 


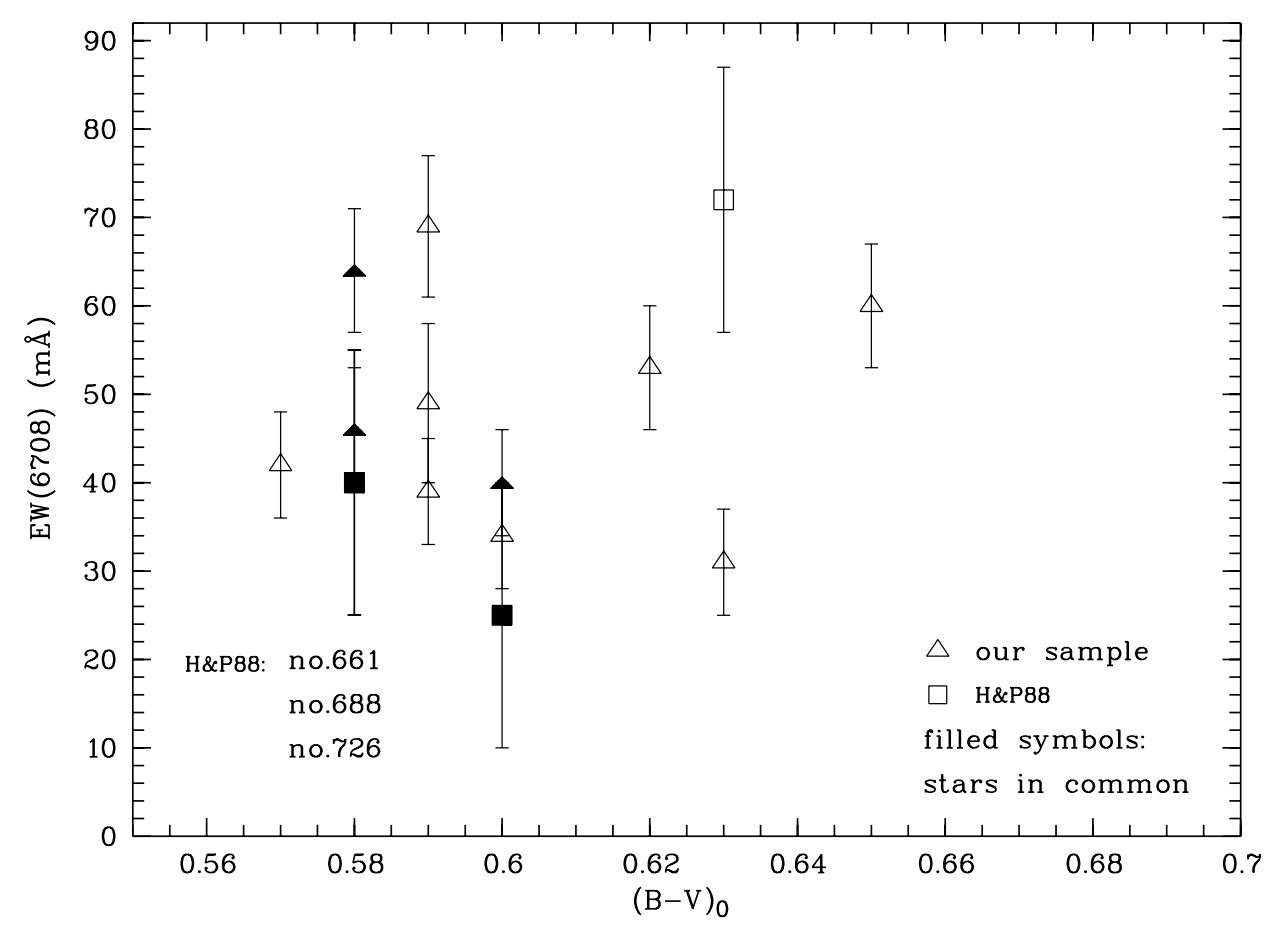

Fig. 2. Li EWs vs. dereddened $B-V$ color for our sample stars (triangles) and the stars in the sample of Hobbs \& Pilachowski (1988 - squares). Filled symbols denote stars in common between the two samples. Note that, as indicated in Table 2, stars no. 661, 688, and 726 in Hobbs \& Pilachowski sample have the same color and Li EW. Of these, star no. 726 is not included in our sample.

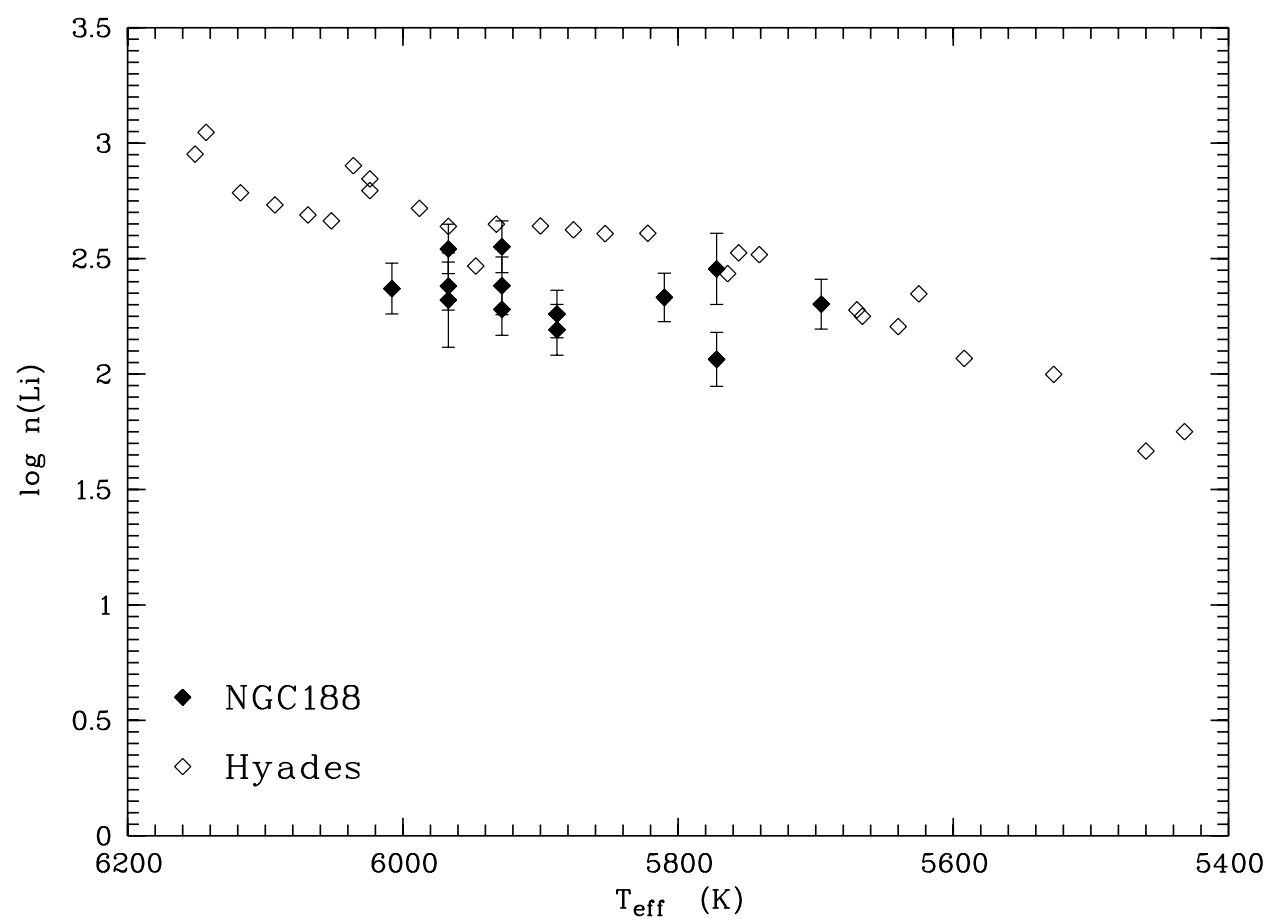

Fig. 3. Li abundances vs. $T_{\text {eff }}$ for the 13 stars in the merged (our + Hobbs \& Pilachowski's) NGC 188 sample (filled symbols) and the Hyades (open symbols).

the solar age cluster M 67, concluded that the mechanism that drives Li depletion appears to stop in the late phases ( $\geq 2 \mathrm{Gyr}$ ) of MS evolution, unless different conditions/parameters lead to different Li depletion timescales. In Fig. 5 we plot the average $\mathrm{Li}$ abundance as a function of age for stars in the $5750-6050 \mathrm{~K}$ temperature range. The Hyades, IC 4651 and NGC 3680, M 67, and NGC 188 are considered in the plot; for M 67 we included only stars lying on the upper envelope $(\log n(\mathrm{Li}) \geq 2)$. Error bars represent $1 \sigma$ standard deviations from the average. The figure evidences a plateau in $\mathrm{Li}$ abundances for ages older than $\sim 2$ Gyr and, except for Li-poor stars in M 67, there seems to be no additional evolution of $\mathrm{Li}$ abundances beyond $2 \mathrm{Gyr}$. 


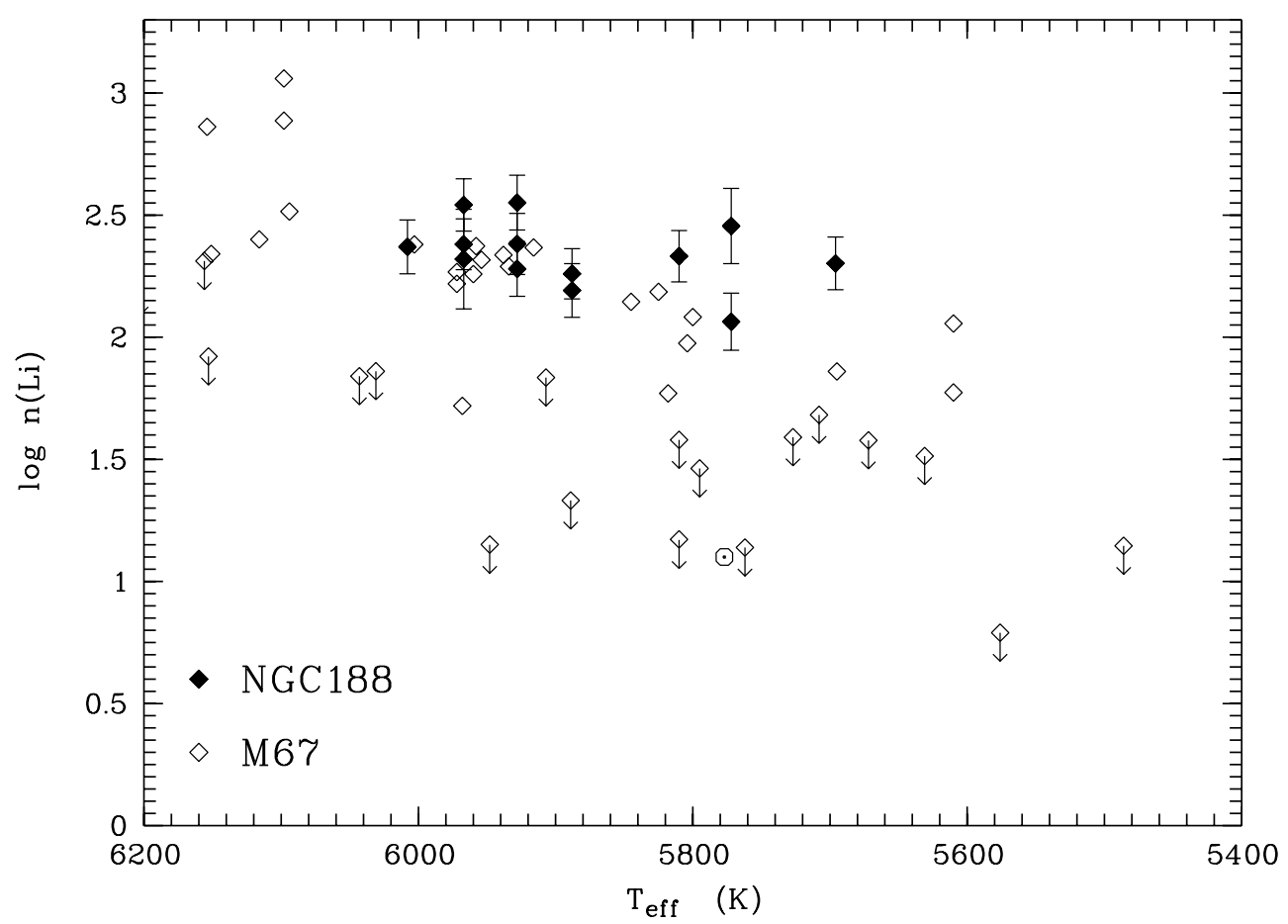

Fig. 4. Same as Fig. 3 but with NGC 188 compared to M 67. The Sun is also shown in the figure.

This result suggests three alternative scenarios: $i$ ) the clusters have all the same initial abundance, but solar-type stars have not undergone additional $\mathrm{Li}$ depletion beyond $\approx 2 \mathrm{Gyr}$, i.e., if a star has not severely depleted $\mathrm{Li}$ at that age, it will not deplete it afterwards (except again for $\mathrm{Li}$-poor stars in $\mathrm{M}$ 67) until first dredge-up and dilution occur; ii) the clusters have all the same initial abundance, but different initial conditions and/or different parameters led to different Li depletion rates, with NGC 188 being characterized by the longest Li depletion timescale. Since on theoretical grounds metallicity, and more in general $\alpha$-element abundances, are thought to affect both standard and non-standard mixing processes (e.g., Chaboyer et al. 1995; Swenson \& Faulkner 1992; Piau \& Turck-Chièze 2002), a difference in chemical composition could be a possible cause for different timescales of Li depletion; iii) the clusters have different initial Li abundances; in particular, the very old cluster NGC 188 might have had, because of its age, a lower Li abundance than the younger clusters. In this case, it must have also undergone considerably less Li depletion than the other clusters to end up with the same average Li abundance.

At present we have no definite clues to discern between these three possibilities; hypothesis $i$ ) is the simplest one, but requires a physical mechanism that is not efficient at ages older than about 2 Gyr. None of the Li depletion processes so far proposed has these characteristics. As to the second scenario, we note that no Li depletion - metallicity relation is found for solar-type stars up to the Hyades age (e.g., Jeffries 2000 and references therein). Furthermore, Pasquini et al. (2001) found $[\mathrm{Fe} / \mathrm{H}]=-0.17 \pm 0.11$ for NGC 3680, while Bragaglia et al. (2001) measured $[\mathrm{Fe} / \mathrm{H}]=+0.16$ for IC 4651 ; the two clusters hence differ in metallicity by a factor of 2 , yet they have the same average Li abundance. This suggests that overall metallicity has little effects on Li depletion also at old ages, at least when the rather narrow range of $[\mathrm{Fe} / \mathrm{H}]$ values covered by Pop. I stars is considered. In any case, our analysis confirms that NGC 188 has solar metallicity and solar metallicity has also been reported for M 67 (e.g., Jones et al. 1999 and discussion therein); thus, even if metallicity would affect $\mathrm{Li}$ depletion, it is not plausible that the two clusters had different Li histories due to different overall metallicities. Besides iron, the abundance of $\alpha$-elements and in particular of oxygen significantly affects stellar opacities, the depth of convective zone, and in principle mixing (e.g., Swenson \& Faulkner 1992; Piau $\&$ Turck-Chièze 2002). With the exception of the Hyades, a detailed $\alpha$-element abundance analysis for the clusters shown in Fig. 5 has so far not been carried out and thus we are not able to investigate whether the flat $\log n(\mathrm{Li})$ vs. age distribution after $2 \mathrm{Gyr}$ is the result of different heavy element abundances; given the old age of NGC 188, it is well possible that the relative abundance of metals in this cluster may differ from the solar distribution. However if $\mathrm{Li}$ evolution in the four clusters of Fig. 5 older than 1 Gyr is driven by heavy element abundances, it would be surprising that these abundances are precisely tuned to give the same average $\mathrm{Li}$ abundance in all clusters. The same argument applies to the third possible scenario; whereas lower initial Li abundance for NGC 188 cannot be excluded (although the inferred solar metallicity of NGC 188 together with the observed Li vs. Fe Galactic enrichment argue against this possibility), this assumption would imply that NGC 188 has also suffered throughout its lifetime a much lower Li depletion than the younger clusters. We concur with Hobbs \& Pilachowski (1988) that the possibility that NGC 188 was characterized by a different initial $\mathrm{Li}$ abundance seems very unlikely. 


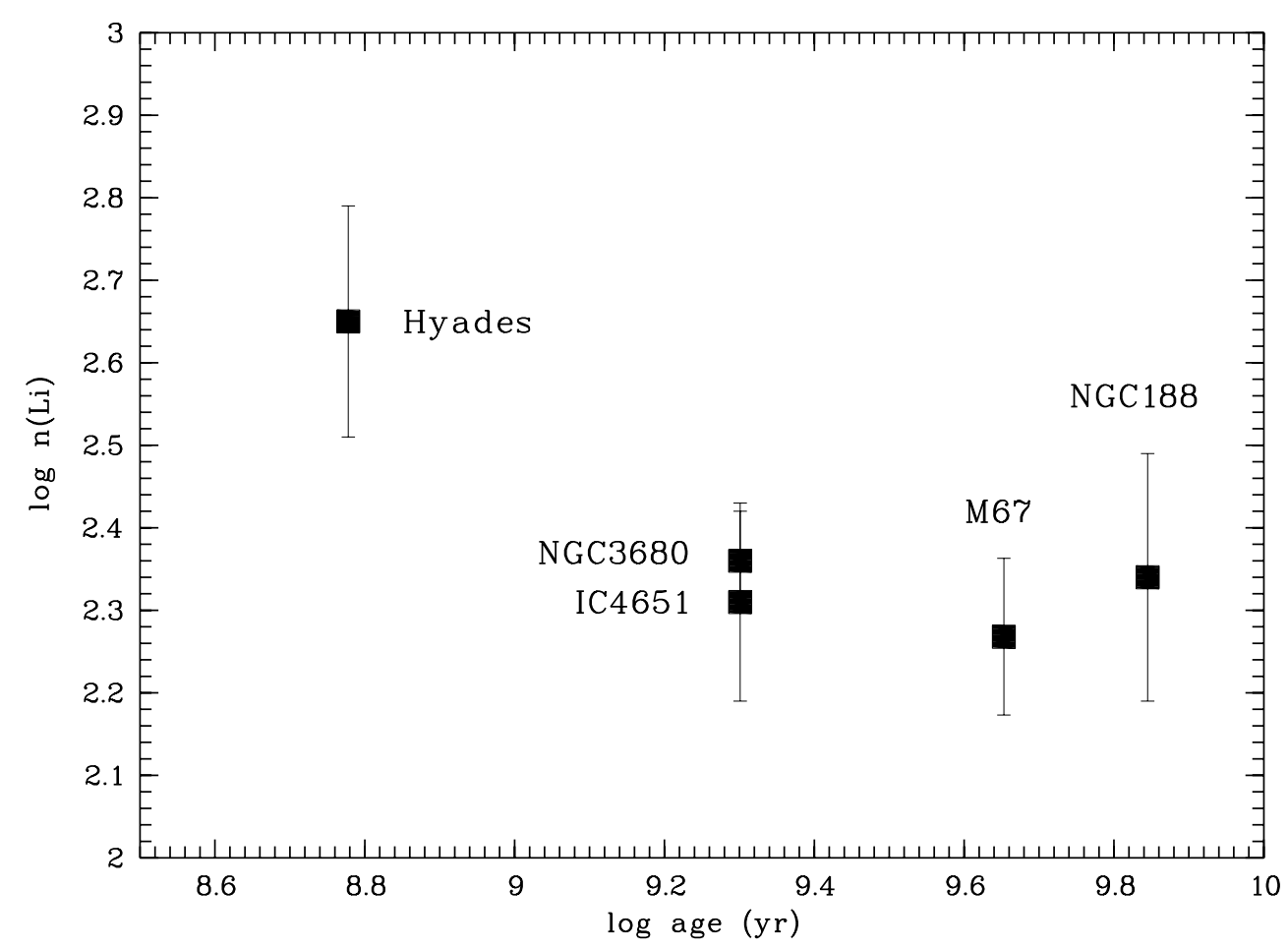

Fig. 5. Average $\mathrm{Li}$ abundance vs. age for five open clusters. The average $\log n(\mathrm{Li})$ values have been computed considering stars in the temperature range $5750 \leq T_{\text {eff }} \leq 6050 \mathrm{~K}$. Error bars denote $1 \sigma$ standard deviations. NGC 752 is not included in the plot since only two stars in the considered $T_{\text {eff }}$ range have available Li measurements. Note however that for these two stars we derive $\mathrm{Li}$ abundances $\log n(\mathrm{Li})=2.32$ and 2.43 (as for the other clusters, we have re-analyzed the original data of Hobbs \& Pilachowski 1986).

As a final remark, we note that the average abundance of the 13 NGC 188 members in our sample is very close to the value of the Li plateau for Pop. II halo stars with $[\mathrm{Fe} / \mathrm{H}] \leq-1.4$ and turn-off stars in globular clusters (e.g., Bonifacio et al. 2002). This point may be a coincidence and Pop. II stars on the Spite's plateau cover a wider range of temperatures; thus, we do not intend to draw any conclusion from it. However, we regard this coincidence as very intriguing and worth of further investigation.

\subsection{The dispersion}

Randich et al. (2000) from the lack of dispersion in Li abundances among solar-type stars of the intermediate age clusters IC 4651 and NGC 3680, concluded that the dispersion must have developed after $\sim 2 \mathrm{Gyr}$; if this is indeed the case, any cluster older than that age should exhibit a dispersion. Our results for NGC 188 suggest instead that M 67 might be a peculiar cluster and that solar-type stars in clusters normally do not develop a dispersion in Li. A larger number of intermediateage/old clusters is obviously needed, as well as new observations of NGC 752, to investigate whether M 67 is really unique and to put this conclusion on firm basis. We recall however that a spread in $\mathrm{Li}$ is also present among old stars in the field (e.g., Duncan 1981; Pallavicini et al. 1987; Pasquini et al. 1994): in particular, we mention that several field stars as old as the Sun, but with much higher Li content exist. The simultaneous presence of Li-rich and Li-poor stars in M 67 and in the field implies that, depending on a parameter that is neither age nor mass, Li destruction can be either rather slow or very fast. Various hypothesis have been proposed in the literature to explain the star-to-star scatter in M 67; for example, the coexistence of two sub-clusters (e.g. García López et al. 1988), a scatter in initial rotation rates (e.g., Jones et al. 1999) or, more recently, a scatter in heavy element abundances (e.g., Piau et al. 2003).

As discussed by Randich et al. (2000), if the dispersion observed in M 67 is due to different initial rotation rates and angular momentum evolutions, the lack of a dispersion in other old clusters and in particular in NGC 188, would imply that solar-type stars in these clusters arrived on the ZAMS with very similar initial rotation rates; this is quite unlikely since a dispersion in initial rotation rates is indeed observed in all the young clusters so far surveyed for rotational periods and/or velocities (e.g., Stauffer et al. 1997; Barnes 2000 and references therein). We also mention that, according to current models including mixing driven by angular momentum, a scatter in $\mathrm{Li}$ abundances at the age of M 67 would imply a scatter in Be abundances. Randich et al. (2002) instead measured the same Be abundances for M 67 stars with a different Li content.

On the other hand, we do not have observational evidences to proof or dis-proof the other two possibilities, i.e., whether the scatter is due to differences in heavy element abundances among M 67 stars or if the cluster results from two different subclusters. We note however that both hypotheses would imply that the population of M 67 is not homogeneous, confirming that M 67 is a peculiar cluster. This would also be in 
agreement with the fact that a dispersion in $\mathrm{Li}$ is observed among field stars, i.e., within a very inhomogeneous sample.

\section{Conclusions}

We have determined $\mathrm{Li}$ abundances for $11 \mathrm{G}$-type stars (with dereddened $B-V$ colors $\left.0.57 \leq(B-V)_{0} \leq 0.65\right)$ in the old open cluster NGC 188. Our data, together with the Li abundances of five stars (three in common with us) previously measured by Hobbs \& Pilachowski (1988), allow us to extend the investigation of $\mathrm{Li}$ depletion in Galactic open clusters to ages 2-4 Gyr older than the Sun. Two are the main results of this study: a) solar-type stars in NGC 188 have all the same Li abundances $(\log n(\mathrm{Li})=2.34 \pm 0.13)$ with virtually no scatter, contrary to what has been observed in M 67; b) solar-type stars in NGC 188 are no more Li depleted than stars in the upper envelope of the $\mathrm{Li}$ abundance vs. temperature distribution of M 67. We have also estimated the cluster metallicity finding $[\mathrm{Fe} / \mathrm{H}] \simeq 0$.

The above results represent further challenges to our interpretation of Li depletion in MS solar-type stars. Based on the results for M 67 and field stars, one would have expected to find a significant fraction of Li-poor stars in NGC 188. Finding that 13 out of 13 members of NGC 188 have a Li abundance only slightly below the Hyades confirms that very old stars are not necessarily Li depleted; at the same time, the absence of NGC 188 members as Li depleted as the Sun or as the most depleted stars in M 67, appears intriguing and raises questions about the possibility of using a single open cluster as truly representative of all clusters of the same age and metallicity.

The comparison of NGC 188 with the upper envelope of M 67, with the 2 Gyr clusters, and with the Hyades shows that the average $\mathrm{Li}$ abundance is virtually the same for clusters with ages $\geq 2$ Gyr. Whereas this result can be due to differences in heavy element abundances leading to different Li depletion timescales for different clusters, this possibility appears to be not very likely. We rather believe that the plateau in $\mathrm{Li}$ abundances observed for ages older than 2 Gyr supports the idea that, for part of the stars, Li depletion may become ineffective beyond this age. A suitable mixing process with these characteristics must be investigated. The spread among M 67 and in the field shows that part of the stars instead undergo a very dramatic additional Li destruction after 2 Gyr. However M 67 remains so far the only old cluster for which a dispersion among solar-type stars has been confirmed. The reasons for this remain unclear, but we suggest that M 67 might represent an inhomogeneous sample in some respect.

We finally mention that the average $\mathrm{Li}$ abundance of NGC 188 is only slightly higher than the Li plateau for Pop. II stars with $[\mathrm{Fe} / \mathrm{H}] \leq-1.4$ in the halo and in globular clusters. Although this may be only a coincidence, it indicates the need of determining Li abundances in other old clusters (both open and globular) in order to link together the Li abundance of Pop. II stars (usually assumed to be equal to the primordial value) with that of Pop. I stars in old open clusters.
Acknowledgements. We thank the referee, Dr. F. Spite, for very useful comments on the manuscript.

\section{References}

Balachandran, S. C. 1995, ApJ, 446, 203

Barnes, S. 2000, in Stellar Clusters and Associations: Convection, Rotation, and Dynamos, ed. R. Pallavicini, G. Micela, \& S. Sciortino, ASP Conf. Ser., 198, 319

Boesgaard, A. M., \& Friel, E. D. 1990, ApJ, 351, 467

Bonifacio, P., Pasquini, L., Spite, F., et al. 2002, A\&A, 390, 91

Bragaglia, A., Tosi, M., Carretta, E., \& Gratton, R. G. 2001, in Cosmic Evolution, ed. E. Vangioni-Flam, R. Ferlet, \& M. Lemoine (World Scientific, Singapore), 209

Carlsson, M., Rutten, R. J., Bruls, J. H. M., \& Shchukina, N. G. 1994, A\&A, 288, 860

Chaboyer, B., Demarque, P., \& Pinsonneault, M. H. 1995, ApJ, 441, 865

Dinescu, D. I., Girard, T. M., van Altena, W. F., Yang, T., \& Lee, Y. 1996, AJ, 111, 1205 (D96)

Duncan, D. K. 1981, ApJ, 248, 651

García López, R. J., Rebolo, R., \& Beckmann, J. E. 1988, PASP, 100, 1489

Gratton, R. G., Bonnano, G., Bruno, P., et al. 2000, Proc. SPIE, 4008, 244

Hobbs, L. M., \& Pilachowski, C. 1986, ApJ, 309, L17

Hobbs, L. M., \& Pilachowski, C. 1988, ApJ, 334, 734

Jeffries, R. J. 2000, in Stellar Clusters and Associations: Convection, Rotation, and Dynamos, ed. R. Pallavicini, G. Micela, \& S. Sciortino, ASP Conf. Ser., 198, 245

Jones, B. F., Fisher, D., \& Soderblom, D. R. 1999, AJ, 117, 330

Kurucz, R. L. 1995, private communication

Pallavicini, R., Cerruti-Sola, M., \& Duncan, D. K. 1987, A\&A, 174, 116

Pasquini, L. 2000, in IAU Symp. 198, The Light Elements and their Evolution, ed. L. da Silva, M. Spite, \& J. R. de Medeiros, 269

Pasquini, L., Liu, Q., \& Pallavicini, R. 1994, A\&A, 287, 191

Pasquini, L., Randich, S., \& Pallavicini, R. 1997, A\&A, 325, 535

Pasquini, L., Randich, S., \& Pallavicini, R. 2001, A\&A, 374, 1017

Piau, L., \& Turck-Chieże, S. 2002, ApJ, 566, 419

Piau, L., Randich, S., \& Palla, F. 2003, in preparation

Randich, S., Pasquini, L., \& Pallavicini, R. 2000, A\&A, 356, L25

Randich, S., Primas, F., Pasquini, L., \& Pallavicini, R. 2002, A\&A, 387,222

Sandage, A. 1962, ApJ, 135, 333

Sarajedini, A., von Hippel, T., Kozhurina-Platais, V., \& Demarque, P. 1999, AJ, 118, 2894 (S99)

Sneden, C. 1973, ApJ, 184, 839

Spite, F., Spite, M., Peterson, R. C., \& Chaffee, F. H., Jr. 1987, A\&A, 171, L8

Soderblom, D. R., Jones, B. F., Balachandran, S., et al. 1993, AJ, 106, 1059

Stauffer, J. R., Hartmann, L. W., Prosser, C. F., et al. 1997, ApJ, 479, 776

Swenson, F. J., \& Faulkner, J. 1992, ApJ, 395, 654

von Hippel, T., \& Sarajedini, A. 1998, AJ, 116, 1789 